\title{
Multidimensional Imaging of Surfaces with Ringing Mode of Atomic Force Microscopy
}

Igor Sokolov ${ }^{1,2}$, Maxim Dokukin ${ }^{2}$

1. Department of Biomedical Engineering, Tufts University, Medford, MA, USA.

2. Department of Mechanical Engineering, Tufts University, Medford, MA, USA.

The ability to measure novel characteristics of samples at the nanoscale opens a new dimension in quantitative study and development of polymers, nanocomposites and biomaterials. Atomic force microscopy (AFM) is a technique which allows simultaneous collecting of multidimensional information of a sample surface. Recently the major AFM manufactures have introduced a novel subresonant tapping imaging mode for AFM operation. This mode has been presented under different names including Digital Pulse Mode ${ }^{\mathrm{TM}}$ (WITec), PeakForce QNM ${ }^{\mathrm{TM}}$ (Bruker), Hybrid mode ${ }^{\mathrm{TM}}$ (NT MDT), QITM mode (JPK), etc. Sub-resonance tapping allows simultaneous collection of four major?? physical channels: height, adhesion, viscoelastic losses, stiffness (there are a few more channels, which however, are just derivatives of the basic four physical channels) [1].

Recently, we introduced a new version of sub-resonance tapping [2,3], in which up to eight new additional physical channels can be recorded in parallel (presently, this mode is commercialized under trade name Ringing mode ${ }^{\mathrm{TM}}$ by NanoScience Solutions, Inc. as an addon attachment to existing AFMs). These channels are

- Pull-off neck height. It is the maximum height of the neck pulled from the sample surface by the AFM probe. It is important to stress that this is a directly measured height, not calculated within some model, see, Fig. 1 for graphical definition of Pull-off neck height.

- Disconnection distance. It is the length of molecules on the sample surface, which are stretched out of the sample by the AFM probe during the pull-off - disconnection event, see, Fig. 1 for graphical definition of Disconnection distance.

- Restored (averaged) adhesion. It is the adhesion restored from the AFM ringing signal. It has much less noise compared to regular adhesion, see, Fig.2a. Due to the lower noise, one can clearly see finer details compared to the regular adhesion (Fig.2b) It is typically less than the regular adhesion. The difference comes from the viscous losses and molecular rupturing during disconnection of the AFM probe from the sample surface or losses due to capillary force between the AFM probe and sample surface.

- Disconnection height. It is the height of the sample when the force acting on the AFM cantilever is equal to the force of restored adhesion.

- Disconnection energy loss. It is the energy losses due to dissipative disconnection of the AFM probe from the sample surface.

- Dynamic creep phase shift. It is the phase difference between the oscillation of the sample or cantilever holder (depending on the AFM model) and AFM cantilever.

- Zero-force height (this channel, though, is available in some commercial AFMs). It is the height of the sample at zero deflection of the cantilever. It produces the height image of the sample with less artifacts due to deformation of the sample by the AFM probe.

- Adhesion height. It is the height of the sample at the maximum negative deflection of the cantilever (when the deflection force is equal to the force of adhesion), see, Fig.2c. It allows to see even small fibers on the sample surface, which otherwise are deformed, and not seen in either 
regular or zero force heights. One can clearly see very fine microridges on the cell surface compared to an "ironed out" regular height image of the same area, Fig.2d. The adhesion height gives much higher spatial resolution compared to the regular and zero force heights.

It should be noted that all these eight channels can be recorded simultaneously with the standard subresonance tapping channels. Moreover, all parameters measured in these additional channels have definite physical meaning, and as such, should be independent on the particular type of instrument used.

Almost all new channels are based on the presumption of existing adhesion between the AFM probe and sample surface. Although adhesion may be zero or insufficiently large in some cases, most of the time the adhesion can be "restored" up to the measurable level by using an AFM probe with larger radius of the apex curvature. For example, a larger radius of the probe is recommended for imaging in water or other liquid environment. Alternatively, the probe can be functionalized with the molecules which can have specific adhesion to the sample surface, or some regions of the surface [4].

\section{References:}

[1] M.E Dokukin and I. Sokolov, Langmuir, 28 (2012) p. 16060.

[2] M.E Dokukin and I. Sokolov, Sci Rep. 7 (2017) p. 11828.

[3] I. Sokolov and M.E Dokukin, Methods in Molecular Biology. ed. Y. Lyubchenko (Springer Series), in press.

[4] I.S. acknowledges funding from NSF grant CMMI 1435655.
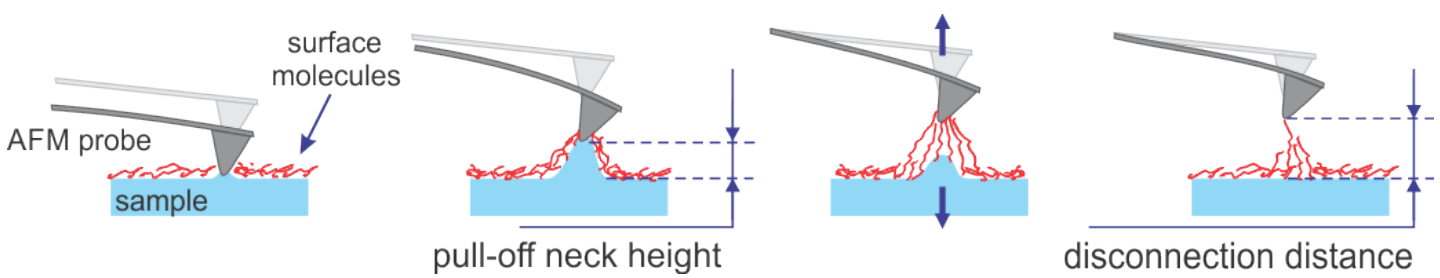

Figure 1. Schematic presentation of definition of two Ringing mode channels, pull off neck height and disconnection distance. Time runs from left to right.

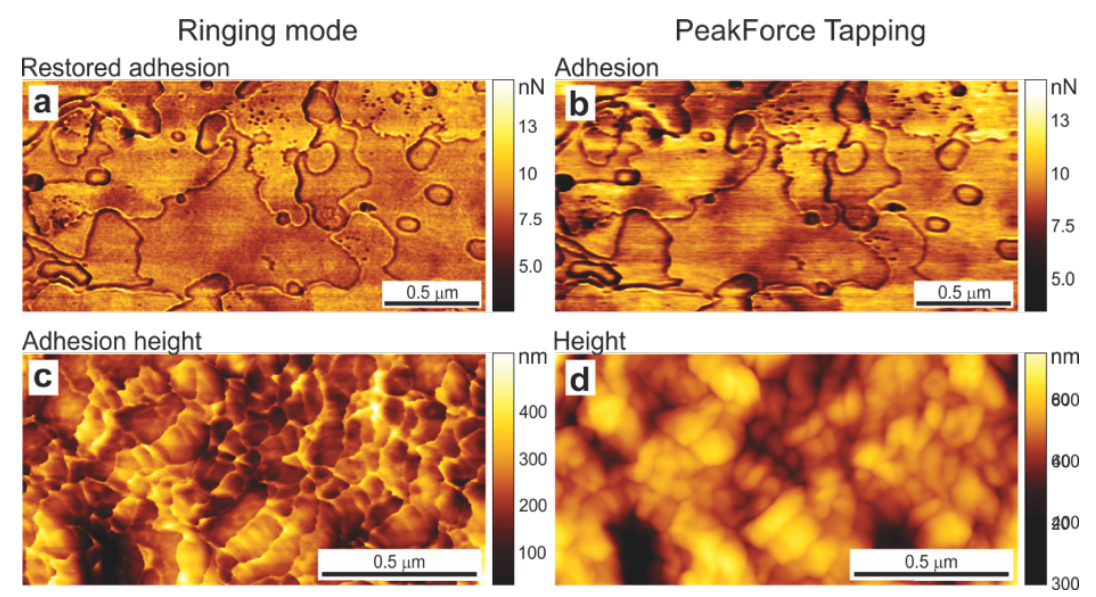

Figure 2. A comparative example of images obtained with Ringing mode and PeakForce tapping. The restored adhesion (a) is compared with the regular adhesion (b) by imaging a skin flake. Adhesion (c) and regular (d) heights are shown for a biological cell. 